\title{
A Rare Cause of Cytopenias in Childhood: Paroxysmal Nocturnal Hemoglobinuria
}

\author{
Baris Yilmaz* \\ Division of Pediatric Hematology and Oncology, Turkey
}

*Corresponding author: Baris Yilmaz, Division of Pediatric Hematology and Oncology, Education Hospital, SB Marmara University Pendik Research, Istanbul, Turkey

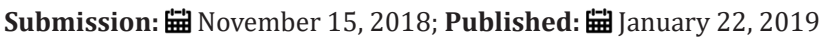

\begin{abstract}
Paroxysmal nocturnal hemoglobinuria (PNH) is a rare hematopoietic stem cell disorder characterized by hemolysis, thrombosis and impaired bone marrow functions. PNH is rarely seen in childhood and usually presents with hypoplastic/aplastic anemia clinic. If the major clinical finding is hemolysis, eculizumab (monoclonal anti-C5 immunoglobulin) can be used for treatment. The other treatment options for PNH are immunosuppressive therapy and stem cell transplantation (SCT). Fluorescent aerolysin (FLAER)-based assay is a high sensitivity test for PNH diagnosis in children. FLAER has become the gold standard for the diagnosis and follow-up of PNH get today.
\end{abstract}

\section{Case Report}

13 year 9-month-old girl was admitted to the emergency service of our hospital with complaints of severe headache, abdominal pain, and vomiting. It was learned that three units of random platelet suspension were given in the hospital. In a detailed anamnesis obtained, it was learned that patient had an increasing complaint of fatigue for a month; the source of her complaint was thought to be linked with the pressure of her upcoming exams and her intensive study schedule.

On physical examination, she had a pale and exhausted look, she was fully conscious and cooperative. In cardiovascular system examination, S1 and S2 sounded normal, there were no additional sounds and murmurs. Heart rate was $90 \mathrm{bpm}$, peripheral pulses were palpated, vein fullness was sufficient. Arterial blood pressure measured as $90 / 55 \mathrm{~mm} / \mathrm{hg}$. Dyspnea and tachypnea were not present, respiratory sounds were normal. Abdominal examination showed increased bowel sounds and diffuse tenderness. There was no organomegaly in the patient. The patient had a severe headache and there was no sign of meningeal irritation.

\section{Laboratory findings}

WBC: $2000 / \mu \mathrm{L}$ (peripheral blood smear formula: $26 \%$ neutrophil, $68 \%$ lymphocyte, $4 \%$ monocyte, $2 \%$ eosinophil), RBC:

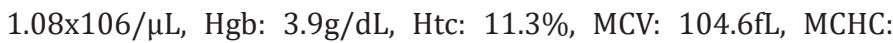
$34.1 \mathrm{~g} / \mathrm{dL}$, platelet count: $69 / \mu \mathrm{L}$ (the value recorded to be $7 / \mu \mathrm{L}$ before transfusion of 3 units platelet suspension in the external center). Atypical cells were not seen in peripheral smear, and platelet clusters of 3-5 cells were seen. Erythrocytes were macrocytic and there was no evidence of hemolysis.
Reticulocyte ratio: $2.89 \%(0.5-2.5)$, corrected reticulocyte ratio: $0.96 \%$, direct Coombs test: negative, ferritin: $219 \mathrm{ng} / \mathrm{mL}$ (7140), folic acid: 4.78ng/mL, vitamin B12: $107 \mathrm{pg} / \mathrm{mL}$ (180-914). The patient's blood glucose level was $107 \mathrm{mg} / \mathrm{dL}$, BUN: $10 \mathrm{mg} / \mathrm{dL}$, creatinine: $0.69 \mathrm{mg} / \mathrm{dL}$, total protein: $6.42 \mathrm{~g} / \mathrm{dL}$, albumin: $4.12 \mathrm{~g} / \mathrm{dL}$, AST: 23U/L, ALT: 6U/L., ALP: 58U/L, LDH: 348U/L (0-248), total bilirubin: $1.78 \mathrm{mg} / \mathrm{dL}$, direct bilirubin: $0.28 \mathrm{mg} / \mathrm{dL}, \mathrm{Na}: 138 \mathrm{mEq} / \mathrm{L}$, $\mathrm{K}: 4 \mathrm{mEq} / \mathrm{L}, \mathrm{Cl}: 106 \mathrm{mEq} / \mathrm{L}, \mathrm{Ca}: 8.8 \mathrm{mg} / \mathrm{dL}, \mathrm{P}: 3.31 \mathrm{mg} / \mathrm{dL}, \mathrm{Mg}:$ $1.99 \mathrm{mg} / \mathrm{dL}$, uric acid: $3.86 \mathrm{mg} / \mathrm{dL}$.

PT: $14.7 \mathrm{sec}$ (11-13), INR: 1.23, aPTT: $21.9 \mathrm{sec}$ (28-36). Hemoglobin electrophoresis: $\mathrm{HbF} 3.7 \%, \mathrm{HbA2}$ : 2\%. Full Urine Assay: Density: 1030, ph: 6.2, Microscopy: Normal.

\section{Hospital course and follow-up}

A severe headache, abdominal pain, and vomiting were diagnosed with pancytopenia. After the diagnosis of pancytopenia in patient who had severe headaches, abdominal pain, and vomiting, the patient admitted to service with preliminary diagnosis of Viral-bacterial infections, meningitis, appendicitis and intraabdominal infections, acute leukemias, aplastic anemia. Blood and urine cultures obtained. In order to exclude central nervous system infections, a lumbar puncture and CSF (cerebrospinal fluid) examination were performed. No bacteria or cells were observed in CSF, there was no growth in CSF culture and the biochemical values of it were normal. a broad spectrum of antibiotic was used in patient's treatment. An abdominal ultrasonography -to exclude possible intraabdominal infections- and chest radiography -to exclude pneumonia- were performed. No pathological findings were 
found in radiological examinations. During the follow-up period, the patient had developed diarrhea and microscopic examination of feces contained no leukocytes and no pathological agents. In feces, Clostridium difficile toxins A and B, Adenovirus, Rotavirus, Giardia and Entamoeba histolytica antigens were screened and resulted to be negative. In blood serological tests, Anti Hbs and Anti CMV IgG were positive and all other serological tests were negative. Parvovirus IgM and IgG and Anti HIV1 / 2 + p24 Ag were negative. During the follow-up period, the patient had significant clinical improvement and blood, CSF, urine cultures remained to be negative (no growth is seen), therefore patient was discharged, and a polyclinic follow-up was scheduled for her.

One week later, she was diagnosed with acute leukemia, aplastic anemia, hypoplastic myelodysplastic syndrome (MDS) though bone marrow aspiration and biopsy. Microscopic examination of bone marrow aspiration with Giemsa staining revealed severe hypocellular bone marrow, which consisted of cells those exhibit uninterrupted maturation. Bone marrow contained each series of cells but displayed predominantly decreased myeloid series (Myeloid / Erythroid series ratio: 1/3). There were dysmorphic changes in the erythroid series. Plastic cell ratio was $<1 \%$. Immunophenotypic analysis of bone marrow aspiration material by flow cytometry revealed lymphocytes in $45 \%$ and non-lymphocyte mononuclear cells in 4\%. Lymphocytes were predominantly composed of $\mathrm{T}$ lymphocytes (29\%). $2 \%$ of the total cells were monocytes and $2 \%$ of all cells were myeloid progenitor cells. In the biopsy report from the pathology, it was learned that the cell / fat ratio was $15 / 85$, there were no ring side oblasts, the dyeable iron level was $<1+$ and the patient was diagnosed with aplastic anemia.

For the differential diagnosis of aplastic anemia, DEB test was performed and resulted as negative, MDS panel (monosomy 5 and 7 , trisomy 5 and 8, 5q-, 7q-) resulted as negative; patient's karyotype was found to be 46XX as expected. By using flow cytometry CD59 expression in erythrocytes, by using FLAER evaluation of macrocytes and granulocytes have been evaluated: PNH clone have been detected in our patient (Table 1).

Table 1: PNH-Clones at diagnosis time.

\begin{tabular}{|c|c|c|c|}
\hline \multicolumn{4}{|c|}{ PNH-Clone } \\
\hline & Type III & Type II & Total \\
\hline Eritrocyte & $0.50 \%$ & $0.30 \%$ & $0.80 \%$ \\
\hline Monocyte & & & $10.50 \%$ \\
\hline Granulocyte & & & $7.80 \%$ \\
\hline
\end{tabular}

Anti-Thymocyte Globulin (ATG, ATGAM®) and cyclosporine treatment were started to treat aplastic anemia. In times of need, supportive therapy was provided with erythrocyte and platelet suspensions. Serum level of cyclosporine measured regularly, and dosage of cyclosporine arranged correspondingly. To determine possible stem cell donors, family members' and patient's HLA tissue typing was done, but no immunologically compatible individual was found. A search for compatible HLA in nationwide and worldwide had been initiated. Because Neutropenia in patient had continued after immunosuppressive therapy G-CSF was added to the treatment plan. After a 1-year follow-up, the patient reapplied to us with sudden developing headache and decrease in sight. Physical examination suggested pseudotumor cerebri. Cranial MRI resulted as normal. Acetazolamide treatment has initiated because pseudotumor thought to be related to cyclosporine treatment. Cyclosporine treatment discontinued and replaced with mycophenolate mofetil (MMF). MMF and G-CSF treatment were stopped at the end of the second year. The patient started to have improved blood cell counts with the exception of platelet numbers, therefore, eltrombopag treatment added to the treatment of the patient. In our patient, there was no need for Eculizumab treatment because the severity of hemolysis was not sufficient to have any clinical effects.

In remission, the patient has been monitored in our clinic, monthly blood counts, PNH clone examination at three-month intervals, annual bone marrow examination have been performed and still carries a low PNH clone.

\section{Discussion}

Paroxysmal nocturnal hemoglobinuria (PNH) is a rare disease in childhood characterized by bone marrow failure, intravascular complement-mediated hemolysis, and thrombosis. This clinical entity which affects precursor hematopoietic cells is the result of an acquired somatic mutation in the phosphatidylinositol-glycanclass A (PIGA) gene [1]. Transcription of PIGA gene produces a glycosylphosphatidylinositol anchor protein (GPI-AP), increase of cells with defective GPI-AP results in uncontrolled complementmediated hemolysis.

The classical triad of $\mathrm{PNH}$ is hemolytic anemia, recurrent thrombosis, and impaired bone marrow functions [2]. These three impairments can be seen in different ratios and severities. Hemolysis may lead to painful crises, severe hemoglobinuria, pulmonary hypertension, and dysphagia or just may be asymptomatic. Thrombosis formations often occur in atypical anatomical localizations. While extremities are less affected, hepatic portal vein (Budd-Chiari), renal veins, mesenteric veins are frequently affected. Bone marrow is also affected in all patients. The most severe form is immune-mediated severe aplastic anemia [3].

PIGA gene is localized on the $\mathrm{X}$ chromosome. In defective synthesis, the levels of CD55 [(decay accelerating factor) accelerates the decay of preformed C3 convertase] and CD59 [(membrane inhibitor of reactive lysis) protects the body from the effects of membrane-attack complexes by downregulating them] are reduced and erythrocytes become susceptible to hemolysis [2,3].

In 2005, International PNH Interest Group in an article they published in Blood magazine has proposed to treat PNH in three different groups and this classification is still being used (Table 2). The first group (classical PNH) is the group with erythroid hyperplasia or minimal changes in the bone marrow with hemolysis and thrombosis. The second group (the group of specific bone marrow diseases) is associated with bone marrow changes 
such as MDS, aplastic anemia and myelofibrosis. Some karyotype anomalies may be found in patients those fall in this category (MDS anomalies such as 5q, 7 and 20q). The third and last group is called Sub-clinical PNH. In this group there are no clinical or laboratory findings that support hemolysis, despite bone marrow failure may accompany [4].

Table 2: Classification of PNH [4].

\section{A. Classic PNH}

B. PNH in the setting of another specified bone marrow disorder (eg. $\mathrm{PNH} /$ aplastic anemia or $\mathrm{PNH} /$ refractory anemia-MDS)

C. PNH-subclinical (PNH-sc) in the setting of another specified bone marrow disorder (eg, PNH-sc/aplastic anemia)

Previously recommended acid-HAM or sucrose-lysis tests for diagnosis are replaced by more sensitive flow cytometric methods and these flow cytometric methods are currently the gold standard in diagnosis. In the FLAER (fluorescently labeled proaerolysin) method, FLAER -a kind of prototoxin for aerolysin-is used in a flow cytometric assay to diagnose PNH. Aerolysin is a GPI-AP-specific bacterial protein because cells affected by PNH lacks normal GPIAP, FLEAR cannot bind PNH affected cells. For leukocytes, GPI-AP can be used to detect defective cells but not for erythrocytes. FITClabeled anti-CD55 and anti-CD59 and anti-glycophorin-A are used for erythrocytes [4,5]. Cells are typed according to their CD55 and CD59 expression rates. Type III PNH cells do not express any CD55CD59, whereas Type II PNH cells express CD55 and CD59 partially, Type I PNH cells express them fully. The presence and severity of hemolysis is directly correlated to the percentage of Type III PNH cells $[5,6]$.

The treatment options should be decided by considering the severity of the disease course. In pediatric $\mathrm{PNH}$, bone marrow deficiency and hypoplastic/aplastic anemia are common, and response to immunosuppressive therapy is quite good. In cases with severe hemolysis, eculizumab (monoclonal anti-C5) treatment can be considered. Hemopoietic stem cell therapy can be tried in patients with MDS / aplastic anemia [7,8].

In this article, we want to emphasize the fact that patients at the border of developing aplastic anemia can be protected from possible future infections and possible need of transfusions though G-CSF and eltrombopag therapies.

\section{References}

1. Zern AED, Symons HJ, Resar LS, Borowitz MJ, Armanios MY, et al. (2014) Detection of paroxysmal nocturnal hemoglobinuria clones to exclude inherited bone marrow failure syndromes. Eur J Haematol 92(6): 467470.

2. Doshi H, Etherington MS, Etherington NB (2017) Case report: paroxysmal nocturnal hemoglobinuria. The Medicine Forum 18: 19-21.

3. Borowitz MJ, Craig FE, Giuseppe JAD, Illingworth AJ, Rosse W, et al. (2010) Guidelines for the diagnosis and monitoring of paroxysmal nocturnal hemoglobinuria and related disorders by flow cytometry. Cytometry Part B (Clinical Cytometry) 78(4): 211-230.

4. Parker C, Omine M, Richards S, Nishimura J, Bessler M, et al. (2005) Diagnosis and management of paroxysmal nocturnal hemoglobinuria. Blood 106(12): 3699-3709.

5. Narita A, Muramatsu H, Sekiya Y, Okuno Y, Sakaguchi H, et al. (2015) Paroxysmal nocturnal hemoglobinuria and telomere length predicts response to immunosuppressive therapy in pediatric aplastic anemia. Haematologica 100(12): 1546-1552.

6. Young NS (2009) Paroxysmal nocturnal hemoglobinuria and myelodysplastic syndromes: clonal expansion of PIG-A-mutant hematopoietic cells in bone marrow failure. Haematologica 94(1): 3-7.

7. Hillmen P, Young NS, Schubert J, Brodsky RA, Socié G, et al. (2006) The complement inhibitor eculizumab in paroxysmal nocturnal hemoglobinuria. N Engl J Med 355(12): 1233-1243.

8. Curran KJ, Kernan NA, Prockop SE, Scaradavou A, Small TN, et al. (2011) Paroxysmal nocturnal hemoglobinuria in pediatric patients. Pediatr Blood Cancer 59(3): 525-529.
Creative Commons Attribution 4.0 International License

For possible submissions Click Here

\section{Submit Article}

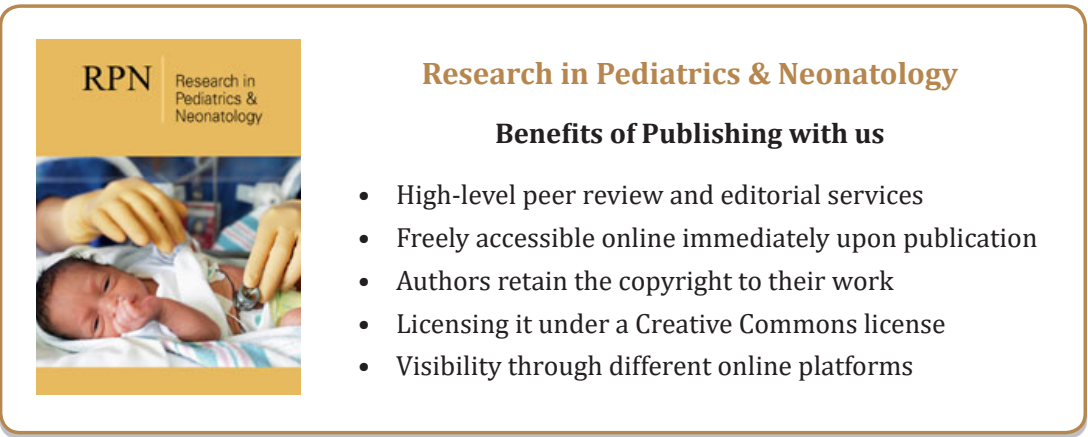

